\title{
Postoperative Complications of Colectomy in Diabetes
}

\section{Patients}

Ramsey T, Giaccio SL and Navarro FA*

Department of General Surgery, University of South

Carolina School of Medicine, USA

*Correspondling author: Fernando A. Navarro, Department of Surgery, Two Medical Park, Suite 306, University of South Carolina School of Medicine, Columbia, SC, 29203, USA

Received: November 20, 2017; Accepted: December 13, 2017; Published: December 20, 2017

\section{Abstract}

Background: In this study, we sought to determine whether Insulin Dependent Diabetes (IDDM) and Non-Insulin Dependent Diabetes (NIDDM) have different risks in comparison to non-diabetics during perioperative period of colectomy using a large national-level database.

Study Design: In our study, de-identified patients who underwent colectomy from 2012 to 2015 were selected from the American College of Surgeons National Surgical Quality Improvement Program (ACS-NSQIP) using the following CPT codes: 44140, 44141,44143, 44144, 44145, 44146, 44147, 44150, 44151, 44155, 44156, 44157, 44158, 44160, 44204, 44205, $44206,44207,44208,44210,44211$, and 44212. Descriptive analysis is used to compare postoperative complication rates in each diabetes group.

Results: IDDM increased the risk of postoperative infection across all studied parameters compared to non-DM. Superficial incision SSI, deep incision SSI, and wound disruption were significantly higher in IDDM compared to non-diabetics (OR $1.39(1.28,1.5), 1.36(1.16,1.60)$, and $1.48(1.27,1.73)$ ) respectively. IDDM was 1.85 times $(1.69,2.03)$ more likely to develop pneumonia and $1.66(1.49,1.85)$ times more likely to develop UTI than non-DM. Sepsis, reoperation, and readmission were also higher in IDDM patients compared to the control with an odds ratio of $1.27(1.17,1.38), 1.36(1.26,1.48), 1.52(1.42$, 1.62) respectively. NIDDM had a smaller increase in risks of superficial incision SSI, pneumonia, UTI, and readmission; however, these risks were not as high as in IDDM.

Conclusion: Clinicians should inform DM patients about their risks with colectomy and encourage them to control their DM, especially to become independent on insulin injection, to avoid these potential perioperative complications.

Keywords: Diabetes; Colectomy; Colorectal surgery; Insulin dependent diabetes; Non-insulin dependent diabetes; Perioperative complications; Perioperative infection; Surgical site infection

\section{Abbreviations}

Non-DM: without Diabetes; IDDM: Insulin Dependent Diabetes; NIDDM: Non-Insulin Dependent Diabetes; SSI: Surgical Site Infection; UTI: Urinary Tract Infection

\section{Introduction}

A careful preoperative evaluation for surgical procedures is the first step in optimizing surgical outcomes, including gathering patients' history and performing physical examination. A risks, benefits, and alternatives analysis is carried out and conveyed to patients so they can make the most informed decision of whether to proceed with the surgery. Therefore, understanding the preoperative risks is extremely important not only in the informed decision making process but also to monitor potential complications.

Diabetes, impacting $9.3 \%$ of the population in the United States, is one of the most common preoperative risk factors for poor surgical outcomes [1]. It impacts multiple organ systems in the body causing gastro paresis, chronic kidney disease, myocardial infarct, retinopathy, and limp amputation. Stress during surgery and general anesthesia causes release of stress-related hormones and inflammatory cytokines, which in turn induce hyperglycemic state by altering insulin regulation, and protein and lipid catabolism [2]. Thus, preexisting diabetes may pose a higher risk of preoperative complications compared to healthy individuals. Multiple studies report a high incidence of preoperative infections associated with poor glycemic control [3-5]. Therefore, preoperative laboratory studies and preoperative glucose control have been carefully evaluated to avoid marked blood sugar, and fluid and electrolyte imbalance.

Tsang S et al. [6] found that the Diabetes Mellitus (DM) was associated with an increased risk of Surgical Site Infection (SSI) (OR 2.04), urinary infection (OR 1.43), and lower respiratory tract infections (OR 1.95) in total hip replacement. Ata A et al. [7] found that the incidence of SSI in patients undergoing colorectal surgery was 3.8 times higher than in patients undergoing general surgery. For those patients undergoing colorectal surgery Insulin Dependent DM (IDDM) and Non-Insulin Dependent DM (NIDDM) were more likely than non-diabetics to develop SSI. For those patients undergoing general surgery, only IDDM were at increased risk.

Despite extensive research on diabetes related infections, there is limited data on the types of wound infections. Data on diabetes's 
Table 1: Patient Demographics for Colectomy from 2012 to 2015.

\begin{tabular}{|c|c|c|c|}
\hline Characteristic & IDDM & NIDDM & Non-DM \\
\hline Median age (years) & 67 & 68 & 61 \\
\hline Weight (pounds) & 194 & 188 & 168 \\
\hline BMI & 32.33 & 31.24 & 27.86 \\
\hline Female & $49 \%$ & $48 \%$ & $53 \%$ \\
\hline
\end{tabular}

Non-DM: without Diabetes; IDDM: Insulin Dependent Diabetes; NIDDM: NonInsulin Dependent Diabetes; Patients over 90 were grouped as 90+; therefore, the median age was used in place of mean; Weight and BMI are averages

effect in cardiac and orthopedic surgeries is abundant, however, similar data surrounding the effect of diabetes on colectomy is lacking. Therefore, we sought to determine whether IDDM and NIDDM have different risks post-colectomy in comparison to non-diabetics using a large national-level database.

\section{Methodology}

\section{Database and study population}

American College of Surgeons National Surgical Quality Improvement Program (ACS-NSQIP) collects preoperative, intraoperative, and 30-day postoperative information on a surgical patient from more than 250 participating hospitals. In our study, deidentified patients who underwent colectomy from 2012 to 2015 were selected from this database using the following CPT codes: 44140, 44141, 44143, 44144, 44145, 44146, 44147, 44150, 44151, 44155, 44156, 44157, 44158, 44160, 44204, 44205, 44206, 44207, 44208, 44210, 44211, and 44212 .

All patients under the age of 18 were excluded. We collected the following variables from 2012 to 2015: diabetes status, age, gender, weight, SSI, UTI, pneumonia, sepsis, readmission, reoperation and length of stay. BMI was collected from 2013 to 2015 since height data for 2012 was not available.

We also collected variables such as indications, oral antibiotics, and postoperative leaks in a participant user file for colectomy separately. This file does not contain CPT codes and diabetes status, so we merged this file with the main file using Case ID as the fixed variable. There are cases listed in the main file that were not found in the colectomy specific file, and some of the CPT codes we used in the main data were not included (44155, 44156, 44157, 44158, 44211,
44212). Therefore, to limit data error, we used the merged data for only indications.

\section{Variable definitions}

Diabetes status is categorized into IDDM, NIDDM and nondiabetes. IDDM are those who require insulin alone or in conjunction with other non-insulin anti-diabetic agents to control their condition. NIDDM are those who take oral on-insulin anti-diabetic agents such as metformin. Patients who have never been diagnosed with diabetes or whose diabetes is controlled with diet and exercise alone are considered non-diabetes.

All postoperative variables must have occurred within 30 days of the procedure. SSI is limited to only postoperative findings and is categorized as superficial, deep, and organs/spaces. Superficial incision SSI involves the skin and subcutaneous tissue, deep incision SSI involves deep soft tissues including fascia and muscles, and organ/ spaces SSI involves any anatomy manipulated during the operations other than incision site. Wound disruption indicates reopening of a previously closed wound. Reoperation indicates a second operation to correct any complications at any surgical facility or hospital within 30 days of postoperative period.

\section{Statistical analysis}

We categorized our CPT filtered data into non-diabetes, NIDDM, and IDDM. In each category, we counted the number of cases and determined their percentage for specified postoperative complications and compared them across diabetic status using chisquare tests. Age was normally distributed but age above 90 was grouped as $90+$, therefore, we opted to use median for the age. Age, weight, BMI, and length of stay are compared using ANOVA tests. Indications in the merged file were analyzed in a similar way.

\section{Results}

A total of 169,325 patients were identified in the NSQIP database that underwent colectomy from 2012 to 2015. A total of 9,130 (5.39\%) patients were IDDM, 14,999 (8.86\%) patients were NIDDM, and $145,196(85.75 \%)$ patients were non-diabetic. The median age of IDDM was 67, NIDDM was 68 , and non-diabetics was 61 (Table 1). There was a $1: 1$ ratio of male to female patients who underwent colectomy despite diabetes status. Patients with IDDM weighed 26

Table 2: Postoperative outcomes in patients underwent colectomy between 2012 and 2015.

\begin{tabular}{|c|c|c|c|c|}
\hline Outcomes & Non-DM & IDDM/Odds ratio* & p value (IDDM) & NIDDM/Odds ratio* \\
\hline Superficial Incisional SSI & $5.49 \%$ & $7.48 \% 1.39(1.28,1.5)$ & $P<0.0001$ & $6.76 \% 1.18(1.10,1.3)$ \\
\hline Deep Incisional SSI & $1.38 \%$ & $1.91 \% 1.36(1.16,1.60)$ & $P<0.0001$ & $1.53 \% 1.09(0.94,1.25)$ \\
\hline Organs/Space SSI & $5.17 \%$ & $5.63 \% 1.09(0.99,1.19)$ & $P=0.0738$ & $4.78 \% 0.94(0.87,1.01)$ \\
\hline Wound Disruption & $1.39 \%$ & $2.09 \% 1.48(1.28,1.73)$ & $P<0.0001$ & $1.30 \% 0.88(0.75,1.02)$ \\
\hline Pneumonia & $3.14 \%$ & $5.75 \% 1.85(1.69,2.03)$ & $P<0.0001$ & $3.78 \% 1.25(1.14,1.36)$ \\
\hline UTI & $2.59 \%$ & $4.27 \% 1.66(1.49,1.85)$ & $P<0.0001$ & $3.13 \% 1.12(1.01,1.24)$ \\
\hline Sepsis & $5.42 \%$ & $6.94 \% 1.27(1.17,1.38)$ & $P<0.0001$ & $4.96 \% 0.90(0.84,0.98)$ \\
\hline Reoperation & $5.58 \%$ & $6.83 \% 1.36(1.26,1.48)$ & $P<0.0001$ & 6.0932 \\
\hline Readmission & $10.04 \%$ & $14.58 \% 1.52(1.42,1.62)$ & $P<0.0001$ & $11.51 \% 1.17(1.10,1.23)$ \\
\hline Ileus & $16.76 \%$ & $21.89 \% 1.40(1.30,1.49)$ & $P<0.0001$ & $17.85 \% 1.08(1.01,1.14)$ \\
\hline
\end{tabular}

Non-DM: without Diabetes; IDDM: Insulin Dependent Diabetes; NIDDM: Non-Insulin Dependent Diabetes; SSI: Surgical Site Infection; UTI: Urinary Tract Infection *Odds Ratio of postoperative outcomes in IDDM or NIDDM in comparison to non-DM patients. 
Table 3: Indications for colectomy procedure from 2012 to 2015.

\begin{tabular}{|c|c|c|c|}
\hline Indications & IDDM & NIDDM & Non-DM \\
\hline Colon cancer & 37.45 & 43.95 & 31.89 \\
\hline Colon cancer with obstruction & 5.2 & 5.04 & 4.8 \\
\hline Non-malignant polyp & 9.51 & 11.07 & 8.4 \\
\hline Chronic diverticular disease & 7.46 & 11.61 & 14.95 \\
\hline Acute diverticulitis & 5.44 & 6.4 & 8.45 \\
\hline Crohn's disease & 1.91 & 1.17 & 6.37 \\
\hline Ulcerative colitis & 1.81 & 0.95 & 2.27 \\
\hline Bleeding & 1.97 & 1.27 & 0.85 \\
\hline Enterocolitis (e.g. C. Difficile) & 1.71 & 0.56 & 0.67 \\
\hline Volvulus & 2.45 & 1.78 & 2.73 \\
\hline Other & 24.91 & 16.03 & 18.43 \\
\hline Unknown & 0.16 & 0.17 & 0.18 \\
\hline Total & 100 & 100 & 100 \\
\hline
\end{tabular}

Non-DM: without Diabetes; IDDM: Insulin Dependent Diabetes; NIDDM: NonInsulin Dependent Diabetes; Indications are expressed as percentages within each diabetes category.

pounds more on average than those without diabetes, while those with NIDDM weighed 20 pounds more on average compared to those without diabetes.BMI was higher in diabetic patients than nondiabetic patients (32.33 in IDDM, 31.24 in NIDDM, and 27.86 in non-diabetes)

IDDM increased the risk of postoperative infection across all studied parameters compared to non-DM (Table 2). Superficial incision SSI, deep incision SSI, and wound disruption were significantly higher in IDDM compared to non-diabetics (OR 1.39 $(1.28,1.5), 1.36(1.16,1.60)$, and $1.48(1.27,1.73))$ respectively. IDDM was 1.83 times more likely to develop pneumonia and 1.65 times more likely to develop UTI than non-DM. Sepsis, reoperation, and readmission were also higher in IDDM patients compared to the control with the odds ratio of $1.27(1.17,1.38), 1.36(1.26,1.48), 1.52$ $(1.42,1.62)$ respectively. NIDDM had a smaller increase in risks of superficial incision SSI, pneumonia, and UTI, and readmission, however, these risks were not as high as in IDDM.

IDDM patients stayed in the hospital 7.5 days on average while patients with NIDDM stayed in the hospital 6.0 days on average; this is slightly higher than the hospital length of stay of non-DM patients with 5.75 days on average. Sepsis was the only statistically significant parameter that appears to be lower in NIDDM than in non-DM.

Colon cancer was the most common indication for colectomy followed by diverticulitis. Interestingly, acute diverticulitis, chronic diverticulitis, and Crohn's disease were more prevalent indicators for colectomy in non-DM than DM patients. Specifically, colectomy for acute diverticulitis was done in $5.44 \%$ of IDDM patients and $8.45 \%$ of non-DM patients. $7.46 \%$ of IDDM patients underwent colectomy for chronic diverticular disease compared to $14.95 \%$ in non-DM patients. Similarly, Crohn's disease as an indication for colectomy was found in $6.37 \%$ of non-DM and $1.91 \%$ of IDDM. In contrast, colon cancer resection was more prevalent in patients with IDDM than in patients without DM. NIDDM fell between IDDM and non-DM group in term of indications (Table 3).

\section{Discussion}

Diabetes is one of the most common chronic medical comorbidities in the United States, with a prevalence of 9.3\% [2]. The diagnosis of this condition causes significant distress on patients in terms of a strict and complicated medication regimen and numerous potential complications, such as cardiovascular incidents, limp amputation, immunosuppression, chronic kidney disease, operative complications, and gastroparesis.DM causes an inflammatory response leading to decreased peripheral blood flow and angiogenesis, which in turn can hinder the healing process of surgical wounds (8). In our study, patients with IDDM had increased risks in developing superficial and deep incision SSI, wound disruption, sepsis, pneumonia, UTI, readmission, reoperation, and longer hospital stay compared to NIDDM and non-DM patients. NIDDM posed a slighter higher risk of superficial incision SSI, pneumonia, UTI, readmission, reoperation, and longer hospital stay. These risks delay patients' recovery, expose them to antibiotics and antibiotics resistance, and additional cost for the hospital stay. Readmission, reoperation, and increased length of stay are postoperative complications that are not only inconvenient to the patients but also incur substantial cost to the healthcare system as a whole. Socially, this means that DM incurs higher medical spending on colectomy than non-DM. In fact, the medical expenditure in diabetic patients is 2.3 times higher compared to non-diabetes patients [9]. It is estimated that diabetes cost the United States 245 billion dollars in 2012 [9]. Therefore, it is extremely important that healthcare providers understand these perioperative outcomes well to educate their patients on the benefits of controlling their DM. Patients do not have to be DM free to get these benefits. Our study found that achieving NIDDM from previously diagnosed IDDM can substantially lower overall perioperative outcomes. Only $5 \%$ of the diabetes is type 1 or juvenile diabetes [10]. By the time of the type 2 diabetes diagnoses, half of the insulin producing cells are destructed, which makes the patients prone to insulin dependent therapy $[11,12]$. According to the American College of Endocrinology and the American Association of Clinical Endocrinologists, patients with A1C greater than $9.0 \%$ or uncontrolled despite oral therapy should be on insulin regimen [13]. Therefore, patients with morbidly uncontrolled DM should target their A1C first to be less than 9.0\%. Its beneficial effect on perioperative outcomes of colectomy will be seen even without the complete resolution of DM.

Gastroparesis is one of the gastrointestinal complications of diabetes, occurring at $1 \%$ in type 2 diabetes and $5 \%$ in type 1 diabetes, and is tightly linked to concurrent obesity and poor glycemic control rather than the duration of the disease [14]. Our study found that IDDM was 1.4 times more likely to develop postoperative ileus compared to non-DM. This risk was not significant in NIDDM. Clinicians should be aware of this finding, and plan to provide the IDDM patients with adequate bowel regimen.

DM was associated with lower colectomy indications for acute diverticulitis, chronic diverticulitis, and Crohn's disease in our study. We have not been able to identify any studies that state that DM is protective or a risk factor for diverticulitis or Crohn's disease. Further research should be done to elucidate this correlation. Interestingly, patients with DM have delayed need for colectomy by 6-7 years in our study. This could imply that DM is in fact protective against 
diverticulitis and Crohn's disease. In contrast, colon cancer indication for colectomy was more common in IDDM patients. This finding is supported by a recent study that type $2 \mathrm{DM}$ is associated with $27 \%$ increased risk of colorectal cancer compared to control [15].

King JT and colleagues [16] found that postoperative glucose control rather than preoperative $\mathrm{A} 1 \mathrm{C}$ and glycemic index affect postoperative outcomes in non-cardiac surgery. In vascular surgery, postoperative hyperglycemia was associated with a higher rate of infection in lower extremity vascular surgery, however, readmission rate was not affected by diabetic status [17]. Sehgal et al. [18] suggests that a tighter glucose control postoperatively (even less than currently recommended guideline of $200 \mathrm{mg} / \mathrm{dl}$ ) would reduce the risk of complications in colorectal surgery and this should be accomplished by intravenous instead of subcutaneous insulin. Our study did not specifically analyze postoperative glycemic control and outcomes; however, patients' preoperative insulin dependent status was correlated with poor postoperative outcomes. This could mean that molecular change in vasculature of chronic DM leads to poor wound healing and impaired immune response postoperatively [8]. Our finding could also imply that patients with DM generally have poor postoperative glycemic control compared to non-DM patients; therefore, they are prone to develop postoperative complications due to postoperative hyperglycemia rather than preoperative DM status. In either case, DM patients have worse outcomes than non-DM.

Our study did have limitations, one of which includes the database of choice. The ACS-NSQIP provides much data about each patient, but does not provide a holistic view of their care. Furthermore, the data only includes participating hospitals. It is likely that smaller, nonacademic hospitals are not included here. Additionally, limitation is inherent in database studies where coding error is of concern. Another limitation of our study includes the unavailability of certain variables from the database, including the missing BMI data for 2012. The merged data did not include all the CPT codes and cases as in the main data sheet. We have not been able to identify the pattern of exclusions for this colectomy specific file. Lastly, we did not have information about patients' $\mathrm{A} 1 \mathrm{C}$, and glycemic control preoperative and postoperatively. This information would further elucidate whether chronic uncontrolled diabetes or poor glycemic control immediately perioperative lead to poor postoperative outcomes, and what level of $\mathrm{A} 1 \mathrm{C}$ or glucose would cause highest rate of complication. Nonetheless, we believe that the data is very valuable in stratifying risk factors of diabetes in colectomy.

\section{Conclusion}

Patients with DM, especially IDDM are more likely to develop postoperative infection, including superficial and deep incision surgical site infection, pneumonia, UTI, and sepsis post-colectomy. They also tend to have reoperation, readmission, postoperative ileus, and longer hospital stay compared to non-DM. Therefore, clinicians should not only inform DM patients about their risks with colectomy but also encourage them to control their DM, especially to become independent on insulin injection, to avoid these potential perioperative complications.

\section{References}

1. Clarke RS, Johnston $\mathrm{H}$, Sheridan $\mathrm{B}$. The influence of anesthesia and surgery on plasma cortisol, insulin and free fatty acids. Br J Anaesth. 1970; 42: 295299.

2. Centers for Disease Control and Prevention. 2016.

3. King JT, Goulet JL, Perkal MF, Rosenthal RA. Glycemic control and infections in patients with diabetes undergoing noncardiac surgery. Ann Surg. 2011; 253: 158-165.

4. Trick WE, Scheckler WE, Tokars JI, Jones KC, Reppen ML, Smith EM, et al. Modifiable risk factors associated with deep sternal site infection after coronary artery bypass grafting. J Thorac Cardiovasc Surg. 2000; 119: 108114.

5. Frisch A, Chandra P, Smiley D, Peng L, Rizzo M, Gatcliffe C, et al. Prevalence and clinical outcome of hyperglycemia in the perioperative period in noncardiac surgery. Diabetes Care. 2010; 33: 1783-1788.

6. Tsang ST, Gaston P. Adverse peri-operative outcomes following elective total hip replacement in diabetes mellitus: a systematic review and met-analysis of cohort studies. Bone Joint J. 2013; 95: 1474-1479.

7. Ata A, Valerian BT, Lee EC, Bestle SL, Elmendorf SL, Stain SC. The effect of diabetes mellitus on surgical site infections after colorectal and noncolorectal general surgical operations. Am Surg. 2010; 76: 697-702.

8. Brem H, Tomic-Canic M. Cellular and molecular basis of wound healing in diabetes. Journal of Clinical Investigation. 2007; 117: 1219-1222.

9. American Diabetes Association. Statistics about Diabetes. 2015.

10. American Diabetes Association. Type 1 Diabetes. 2017

11. Gastaldelli A, Ferrannini E, Miyazaki Y, Matsuda M, Defronzo RA. Beta-cel dysfunction and glucose intolerance: results from the San Antonio metabolism (SAM) study. Diabetologia. 2004; 47: 31-39.

12. U.K. prospective diabetes study 16. Overview of 6 years' therapy of type II diabetes: a progressive disease. U.K. Prospective Diabetes Study Group. Diabetes. 1995; 44: 1249-1258.

13. Jellinger PS, Davidson JA, Blonde L, Einhorn D, Grunberger G, Handelsman $Y$, et al. Road maps to achieve glycemic control in type 2 diabetes mellitus: ACE/AACE Diabetes Road Map Task Force. Endocr Pract. 2007; 13: 260 268.

14. Bharucha AE. Epidemiology and natural history of gastroparesis. Gastroenterol Clin North Am. 2015; 44: 9-19.

15. González N, Prieto I, Del puerto-nevado L, Portal-Nuñez S, Ardura JA, Corton M, et al. 2017 update on the relationship between diabetes and colorectal cancer: epidemiology, potential molecular mechanisms and therapeutic implications. Oncotarget. 2017; 8: 18456-18485.

16. King JT, Goulet JL, Perkal MF, Rosenthal RA. Glycemic control and infections in patients with diabetes undergoing noncardiac surgery. Ann Surg. 2011; 253: 158-165.

17. Vogel TR, Smith JB, Kruse RL. The association of postoperative glycemic control and lower extremity procedure outcomes. Journal of Vascular Surgery. 2017; 66: 1123-1132.

18. Sehgal R, Berg A, Figueroa R, Poritz LS, McKenna KJ, Stewart DB, et al. Risk factors for surgical site infections after colorectal resection in diabetic patients. J Am Coll Surg. 2011; 212: 29-34
Austin J Surg - Volume 4 Issue 4 - 2017

ISSN : 2381-9030 | www.austinpublishinggroup.com

Navarro et al. (C) All rights are reserved
Citation: Ramsey T, Giaccio SL and Navarro FA. Postoperative Complications of Colectomy in Diabetes Patients. Austin J Surg. 2017; 4(4): 1111. 